Erzsébet Knipf - Márta Müller (Budapest):

\title{
Mehrsprachigkeit im Spiegel der deutsch-ungarischen Lexikografie um die Jahrhundertwende (19./20. Jh.)
}

\section{Einführung}

Im vorliegenden Beitrag gedenken wir unserer Kollegin, der hervorragenden Lexikografin für das Sprachenpaar Niederländisch-Ungarisch, Frau Professor Elisabeth Mollay, indem wir einen Einblick in die Entwicklung und den Stand der zweisprachigen lexikografischen Praxis in Ungarn in den Jahrzehnten der Jahrhundertwende vom 19. zum 20. Jh. geben. Da es um ein interdisziplinäres und weit verzweigtes Thema geht (Geschichte, Kulturgeschichte, Sprachwissenschaft, Soziologie), das hier nicht in seiner vollen Breite ausgeführt werden kann, muss zeitlich und die kulturhistorischen Ereignisse betreffend weit ausgeholt werden. Die Lage, der Gebrauch der Sprache(n) durch die Bevölkerung des betreffenden Landes, hier: eines multi-ethnischen und multi-lingualen Reiches ist immer eingebettet in die Umgebung und weitgehend abhängig von den politischen, wirtschaftlichen und sozialen Geschehnissen und Umständen, die sich in einer bestimmten Zeit in den Regionen ereignen.

Daher soll eingangs ein kurzer, historischer Überblick über die umstrittene Sprachenfrage, über die Gestaltung und die Veränderungen im politischen Umfeld sowie in der Sprachenpolitik der an der Regierung Beteiligten in der Monarchie ab Ende des 18. Jahrhunderts gegeben werden. Damit eng verknüpft werden einzelne ausgewählte Etappen der nationalen Bestrebungen des Königreichs Ungarn um die Etablierung der ungarischen Sprache, resp. die Zeit der Spracherneuerung in Ungarn, die als Folge der Erstarkung des Nationalbewusstseins in Ungarn entstanden ist, skizziert. Mit der Sprachreform gingen ein kultureller, literarischer Aufschwung sowie maßgebend auch das Aufblühen der ungarisch-deutschen Lexikografie in Ungarn einher. Im Hauptteil dieses Aufsatzes wollen wir unser Augenmerk auf ein in der Fachliteratur eher vernachlässigtes kulturhistorisch-sprachliches Thema, auf die Behandlung der Mehrsprachigkeit durch die zweisprachige Wörterbuchkultur in Ungarn an der Wende des 19. und 20. Jahrhunderts aus metalexikografischer Sicht richten, d.h. an welchen Stellen der Makro- und Mikrostruktur die äußere und innere Mehrsprachigkeit in dem deutsch-ungarischen Wörterbuchteil von Simonyi / Balassa (im Weiteren: SBDU, 1899) sowie von Kelemen (im Weiteren: KGHDU, 1914) erscheint. ${ }^{1}$

1 Die Kenntnis zweier verschiedener, voll ausgebauter Kultursprachen nennt man äußere Mehrsprachigkeit, dahingegen die Kenntnis zweier Varietäten derselben Gemeinsprache innere Mehrsprachigkeit (Riehl 2014: 16-17). Dieser Beitrag untersucht also die 


\section{Zum sprachenpolitischen Hintergrund der Epoche}

Das Königreich Ungarn war in der Zeit der Monarchie im wahrsten Sinne des Wortes ein Vielvölkerreich mit den - auf seinem Territorium lebenden und gesprochenen - vielen kleinen Sprachen und vielsprachigen Minderheiten, jedoch ohne eine im juristischen Sinne etablierte Mehrheitssprache und zudem auch mit einem recht unterschiedlichen Prestige der vielen im Land gesprochenen kleinen Sprachen. Als eine symbolische Klammer galt das über den vielen kleinen gesprochenen Sprachen stehende Latein, als eine der gültigen Verwaltungs- und Regierungssprachen, die auch von den Habsburgern als solche mehrfach bestätigt wurde, doch auch deren Prestige fing an zu bröckeln, man begann sich davon zu distanzieren und diese Leerstelle musste dann aus sprachpolitischer Sicht gefüllt werden. Die Sprachenfrage der Monarchie flammte im gesamten Reich immer wieder auf und geriet häufig in den Mittelpunkt von politischen Debatten und Unruhen, mit all ihren Folgen und Nachwirkungen in allen Bereichen des Alltags, für fast alle Bevölkerungsschichten der verschiedenen Nationalitäten.

Die Sprachenpolitik der Habsburgermonarchie stellt selbst ein großes Forschungsprojekt dar, auch zur Erörterung der Entstehung der zweisprachigen Lexikografie in Ungarn müssen einige wichtige Vorläufer und Meilensteine aus einer kulturhistorischen und soziolinguistischen Sicht kurz zusammengefasst werden. So werden wir anhand einiger Eckpunkte die Sprachenpolitik unter der Herrschaft von Maria Theresia und Joseph II., die Sprachentradition in der Bildungspolitik der Monarchie kurz andeuten, die Ungarische Spracherneuerungsbewegung erwähnen und deren Früchte kurz vorstellen. Dieser Hintergrund sowie weitere wichtige kulturpolitische Ereignisse sind als Vorläufer zum Aufblühen der damals in Entstehung begriffenen zweisprachigen Lexikografie in Ungarn zu betrachten.

Die Kaiserin Maria Theresia strebte vor dem Hintergrund der überaus großen, im wahrsten Sinne des Wortes gelebten Sprachenvielfalt ihres Reiches eine bestimmte, im allgemeinen Sinne des Wortes verstandene Mehrsprachigkeit an. Zur Aufrechterhaltung der Kommunikation zwischen Zentrum und Peripherie ihres großen Reiches sowie zum Zweck des Funktionierens der zentralen Verwaltung in allen nahen und weit entfernten Regionen war eine ausgleichende Förderung und Sicherung der Verwendung aller in der Monarchie anerkannten und gesprochenen Sprachen angesagt. ${ }^{2}$ Gleichzeitig entstand z.B. auch die

ausgewählten Wörterbücher darauf hin, wie sie mit den (meistens diatopischen) Varietäten des Deutschen bzw. mit den Fremdwörtern umgehen.

2 Der Verfassungsentwurf aus dem Jahre 1848 sicherte allen Nationalitäten der Monarchie die Gleichberechtigung aller landesüblichen Sprachen in Schule, Amt und öffentlichen Leben. Dieses Nationalitätenrecht wurde auch vom Staatsgrundgesetz vom 21. Dezember 1867 übernommen. Artikel 19. des Staatsgrundgesetzes vom 21. Dezember 1867, „über die allgemeinen Rechte der Staatsbürger für die im Reichsrathe vertretenen Königreiche und Länder“ legt Folgendes fest: „Alle Volksstämme des Staates sind gleichberechtigt, und jeder 
Privilegierung einiger weniger Sprachen (wie Deutsch und Latein) durch die Verwaltungspraxis und durch die Volksbildung (vgl. Haslinger 2008: 84ff.). Das elaborierte Deutsch verkörperte in höheren sozialen Schichten ein prestigeträchtiges Symbol, es verkörperte im ganzen Reich die Sprache der Modernität, galt als die wichtigste Voraussetzung des Fortschritts, der Entwicklung der Wirtschaft, der Kultur und der Mode und galt als Vorbild für alle anderen im Reich beheimateten sprachlichen und ethnischen Minderheiten. So bestand auch eine gewisse Erwartungshaltung gegenüber den sozial höheren Schichten, die deutsche Sprache gut zu beherrschen, im Alltag, in der Verwaltung, bei den Behörden mehrsprachig agieren zu können und dass gerade durch die VertreterInnen dieser Schicht auch eine Loyalität gegenüber der deutschen Sprache im Reich aufgebaut und sichtbar gemacht werde. Andererseits war auch die Bestrebung sichtbar, dass die Sprachen der jeweiligen Regionen von den lokalen Vertretern der Zentralstaatlichkeit erlernt und beherrscht werden sollen. In den vielsprachigen ländlichen Bereichen des Reiches, in sozial niedrigeren Schichten setzte sich jedoch in der Alltagskommunikation eine natürliche gesprochene Mehrsprachigkeit durch, da die zwischenmenschlichen Interaktionen unter Menschen verschiedener Muttersprachen durch natürliche Sprachkontakte vollzogen, d.h. höchstwahrscheinlich mehrsprachig abgewickelt wurden.

Im Mittelpunt der Politik von Joseph II. stand eine allgemeine Stärkung und Verbreitung der deutschen Sprache (eine sog. „Germanisierungspolitik“), Deutsch wurde in seiner Funktion als eine unifizierende Sprache dargestellt. Auch die 1777 eingeleitete Schulreform „Ratio Educationis“ widerspiegelte eindeutig dieses Bestreben. Zwischen 1784-1790 wurde im Königreich Ungarn und in Siebenbürgen Deutsch als Pflichtsprache, d.h. als Verwaltungs- und Unterrichtsprache verbindlich eingeführt, selbst ohne Rücksichtnahme auf die nationalen Traditionen und Wünsche der vielen in diesen Regionen lebenden Völker (vgl. Haslinger 2008: 92). Ein Hofkammerdekret aus dem Jahre 1822 verlangte sogar, dass allen in die zentrale Verwaltung gelangten nicht deutschsprachigen Urkunden eine deutsche Übersetzung beigefügt werden musste. In größeren Städten des Reiches genoss die deutsche Sprache im Kulturund Wirtschaftsleben überall ein hohes Prestige, unterstützt und vor allem getragen durch den höheren sozialen Status der in diesen Bereichen beschäftigten Bevölkerungsschichten. Ab der zweiten Hälfte des 18. Jahrhunderts hatte der

Volksstamm hat ein unverletzliches Recht auf Wahrung und Pflege seiner Nationalität und Sprache. Die Gleichberechtigung aller landesüblichen Sprachen in Schule, Amt und öffentlichem Leben wird vom Staate anerkannt. In den Ländern, in welchen mehrere Volksstämme wohnen, sollen die öffentlichen Unterrichtsanstalten derart eingerichtet sein, daß ohne Anwendung eines Zwanges zur Erlernung einer zweiten Landessprache jeder dieser Volksstämme die erforderlichen Mittel zur Ausbildung in seiner Sprache erhält.“ 
Wiener Hof die alleinige Vermittlerrolle der westlichen Kultur nicht nur im Reich, sondern auch in Ungarn übernommen. ${ }^{3}$

Doch die starke Germanisierungspolitik von Joseph II., eine fehlende Rücksichtnahme auf die vielen Völker und deren Sprachen, auf die sprachlichkulturelle Pluralität des Reiches führten u.a. zu einer Reihe von Kettenreaktionen unter diesen Völkern. Auch die damals entstandene außenpolitische Situation, z.B. eine drohende Türkengefahr, das erneute intensive nationale Erwachen insbesondere bei den ungarischen Ständen, deren Nationalbewusstsein in dieser Zeit erstarkte und noch weitere soziale und innenpolitische Faktoren führten dazu, dass ab 1792 an den Schulen auch Ungarisch zum Pflichtfach wurde, trotz der Tatsache, dass Ungarisch damals noch nicht standardisiert war. Es musste ein langer Weg beschritten und viele Hürden überwunden werden, bis Ungarisch dann 1844 offiziell vom ungarischen Landtag zur Amtssprache erklärt wurde.

\section{Zur damaligen Lage der ungarischen Sprache}

Bereits während der 150 Jahre langen Dreiteilung des Landes (1541-1720) verfolgten die einzelnen ungarischen Landesteile völlig unterschiedliche Sprachpraktiken: Während in der Westhälfte, welches Gebiet zum katholischen Habsburg gehörte, die Sprachen des Wiener Hofes, d.h. Deutsch, Italienisch, Französisch und Latein die gebräuchlichen Sprachen waren, wurde im mittleren Teil, das zum osmanischen Reich gehörte, Ungarisch und Türkisch verwendet, Ungarisch auch deshalb, weil in diesem Landesteil die Mehrheit der protestantischen Gelehrten lebte, obgleich sie an deutschen Universitäten studierten und ein gutes Deutsch sprachen ${ }^{4}$, doch war ihr nationales Bewusstsein stärker. Der östliche Teil (Ostungarn und Siebenbürgen) unterstand zwar auch den Türken, doch als ein „Satrapenstaat“ behielten sie ihre ungarische Sprache als Hauptsprache im Alltag und in Publikationen.

Die Grundlagen und Triebkräfte des Aufschwungs einer zweisprachigen Lexikografie in der untersuchten Epoche in Ungarn beruhen - wie bei allen Nationen und Ländern - einerseits auf soziohistorischen Ereignissen, Begebenheiten, die sich vor allem auf politischer Ebene in der Monarchie niederschlugen, andererseits auch auf einer Reihe von makro-soziolinguistischen Umständen, wie der Sprachideologie und vor allem der Sprachenpolitik, die den Zustand, das Entwicklungsniveau sowie die Verbreitung und den Gebrauch der ungarischen (und anderer Sprachen im Reich) bestimmt haben.

Der Beginn des Standardisierungsprozesses des Ungarischen wird auf die Zeit nach 1772, auf den Beginn der Aufklärung in Ungarn, gelegt.

3 Das war die Zeit, als viele Fremdwörter französischer, italienischer Provenienz in die ungarische Sprache gelangten.

4 Für ihre religiöse Zugehörigkeit gab es keine entsprechenden Ausbildugsmöglichkeiten in Österreich. 
Wie oben angedeutet, rief die strenge Germanisierungspolitik Josef II., die zwar am Totenbett des Kaisers zurückgenommen wurde, im Königreich Ungarn heftige Reaktionen hervor. Doch auch in anderen Regionen der Monarchie kam es zu eindeutigen Forderungen der Souveränität im Sinne des nation-buildingProzesseses, es wurden sprachpolitische Forderungen von den einzelnen Ethnien formuliert, die eine Aufwertung der eigenen, nichtdeutschen Landessprache forderten (Näheres zu den betroffenen Ethnien und Sprachen s. bei Haslinger 2008: 90 ff.).

Die in Ungarn eingesetzte Spracherneuerungsbestrebung, einer der wichtigsten Meilensteine des Standardisierungsprozesses des Ungarischen, entstand zunächst nicht in den großen städtischen, kulturellen Zentren, sondern in der Peripherie des Landes, im Kreise einiger sehr mutigen und begeisterten Gelehrten, unter ihnen Wissenschaftler, Politiker, Publizisten, geleitet von Ferenc Kazinczy, dem Gymnasiallehrer. Diese Bewegung verbreitete sich bald und fand viele Nachfolger, die sich als Sprachschöpfer, als Wortbildungsexperten, als Sprachregler (heute würde man sie Sprachnormierer nennen), Stilerneuerer, Sprachkritiker hervorgetan haben, um die ungarische Sprache zu modernisieren und sie als die Sprache des Landes, als Sprache der Verwaltung und Bildung in Ungarn zu erheben. Dieser langwierige Standardisierungsprozess der Sprache wurde weitgehend unterstützt und gefördert durch eine Reihe kulturhistorischer Ereignisse: 1832 erschien die „Erste Ungarische Rechtschreibung“ (Magyar helyesírás és szóragasztás főbb szabályai), 1847 erschien die „Erste Ungarische Grammatik“ von den Autoren Czuczor / Vörösmarty (Kárpáti 2003: 133) und nicht zuletzt muss einer der wichtigsten Höhepunkte erwähnt werden: 1825 die Gründung der Ungarischen Akademie der Wissenschaften von István Széchenyi, die als Symbol, gleichzeitig als Hauptsitz und Bewahrer der ungarischen Sprache betrachtet wurde.

Es war ein langer und mühsamer Weg, doch endlich wurde auf dem Landtag 1844 die ungarische Sprache als Amtssprache angenommen.

Als Folge dieses großen Aufschwungs und zur Etablierung des Ungarischen als normierte, standardisierte Sprache erschienen in dieser Zeit mehr als 100 Wörterbücher, die zweisprachig (mit den Sprachenpaaren Deutsch und Latein) sowie auch einsprachig waren (Regional-, Fremd-, Kinder-, Dialektwörterbücher etc.) (hinsichtlich des Sprachenpaares Deutsch-Ungarisch und vice versa vgl. Fejér 1995: 214-219).

In der lexikografischen Praxis standen somit viele wichtige und große Aufgaben: die einzelnen bislang entlehnten Wortschätze auf ihre Herkunft und Bedeutung hin zu durchforsten, zu beschreiben, (z.B. auch die durch die Doppelmonarchie in die Verwaltung vom Wiener Hof entlehnten Lemmata) ins Ungarische einzubürgern, die einzelnen Kriterien der Entlehnungen festzuhalten, die grammatischen Formen der standardisierten Flexionsparadigmen darzustellen. Doch vor allem und in erster Linie war das Ziel, eine sprachliche Transparenz in der Denkweise und Kultur in zweisprachigen Wörterbüchern in 
beiden Sprachen zu erreichen, das Kennenlernen, das Erlernen und die Verbreitung der gereinigten und normierten ungarischen Sprache zu fördern, damit neben Deutsch, Latein, Französisch nun auch Ungarisch als Sprache der „Unterhaltung“ und zum Aufbau eines Sprachbewusstseins und einer nationalen Identität der ungarischsprachigen Bevölkerung beitragen konnte.

In den nächsten Kapiteln wird vorgestellt, inwiefern diese auch in Ungarn erund gelebte Mehrsprachigkeit in Bezug auf die Präsenz und Markierung von Fremdwörtern und diatopisch/national diversen Varietäten des Deutschen in den deutsch-ungarischen Wörterbüchern um die Jahrhundertwende Eingang gefunden hat.

\section{Allgemeines über die untersuchten deutsch-ungarischen Wörterbücher}

Das deutsch-ungarische Wörterbuch von Simonyi / Balassa (1899) beinhaltet circa 50.000 Lemmata. Zwar wird darauf hingewiesen, dass es sowohl ungarische als auch deutsche Benutzer berücksichtigt, doch wurde es als passives Wörterbuch für Ungarn, für die Herübersetzung aus dem Deutschen ins Ungarische geschrieben. Seine Stichwortliste ist striktalphabetisch geordnet, mit nestbildenden Abweichungen (vgl. Fejér 1995: 35), letztere ermöglichen die Übersicht jener Stichwörter, welche mit derselben ersten Konstituente gebildet sind. Flektierte Wortformen (z.B. Präteritumformen von starken Verben, Suppletivformen von Adjektiven, Pronomina) erhielten bei Simonyi / Balassa einen selbständigen Lemmastatus (vgl. Abb. 1).

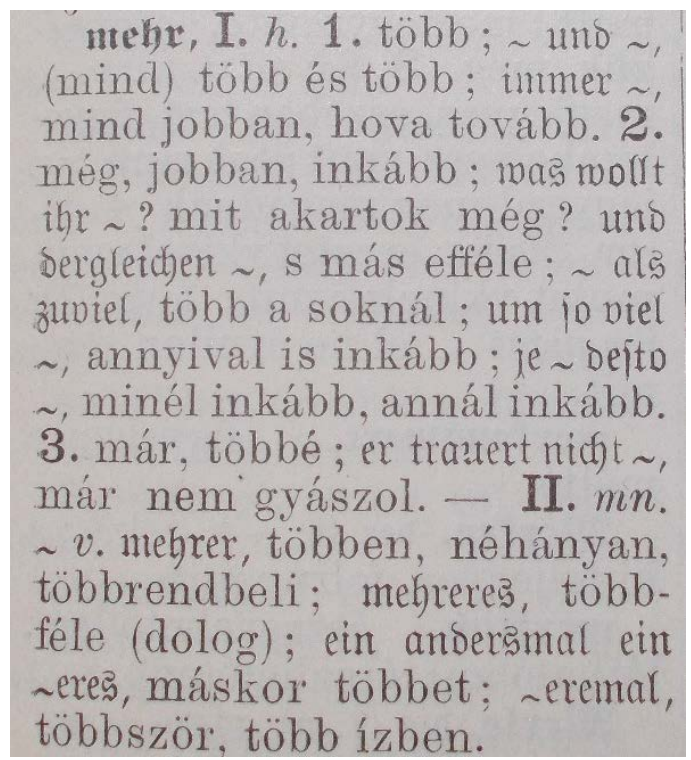

Abbildung 1: mehr als selbständiges Stichwort im SBDU

Für die fachmännische Beschaffenheit des Wörterbuchs bürgt die philologische Qualifikation der Redakteure (beide waren Sprachwissenschaftler). 
Kelemens deutsch-ungarisches Wörterbuch (1914) enthält genauso wie das SBDU ungefähr 50.000, striktalphabetisch geordnete Stichwörter. In ihm finden sich keine Hinweise auf anvisierte Benutzergruppen, doch aus dem Aufbau der Mikrostrukturen kann man darauf schließen, dass es ebenfalls als passives Wörterbuch für Ungarn konzipiert wurde. Komposita, Ableitungen, Homonyme haben einen selbständigen Lemmastatus, flektierte Wortformen werden im KGHDU als Verweislemmata gebracht, deren Anfang mit einem Asteriskus gekennzeichnet wird (vgl. Abb. 2).

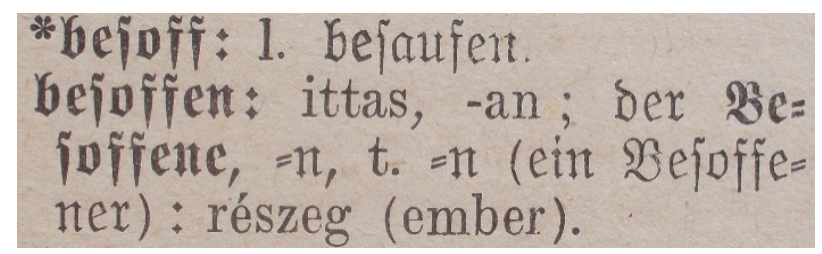

Abbildung 2: Die flektierte Wortform *besoff als Verweislemmata im KGHDU

Ebenfalls als Verweislemmata gelten jene Stichwörter, von denen aus auf ihre orthographischen Varianten verwiesen wird. Die Angabe der Verweislemmata von orthographischen Varianten wird mit einer eckigen Klammer angesetzt (vgl. Abb. 3).

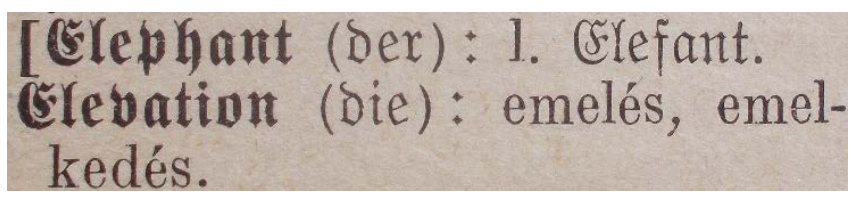

Abbildung 3: Beispielartikel [Elephant für den Verweis auf eine orthographische Variante im KGHDU

Im Vergleich zu der lexikografischen Praxis des philologisch nach deutschem Vorbild geschulten Simonyi, schob Kelemen in dem KGHDU Infokasten zwischen die Wörterbuchartikel, die aus dem, dem Infokasten vorangehenden Artikel ausgelagerte, wesentliche, doch nicht essentielle Informationen über die Morphosyntax, Orthographie, Wortbildung bzw. Pragmatik des vorangehenden Lemmas darstellen (vgl. Abb. 4 sowie 5).

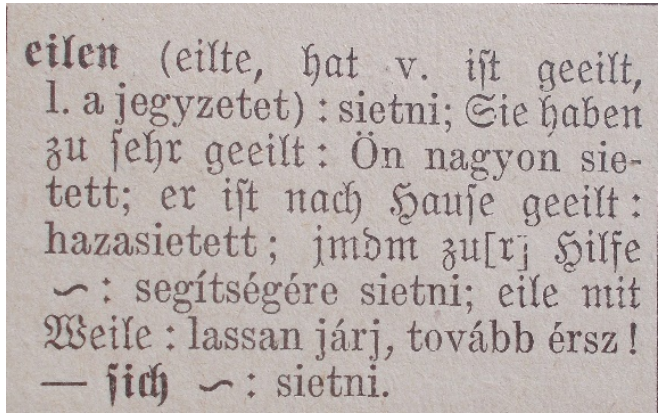

Abbildung 4: eilen, h. bzw. i. im KGHDU 


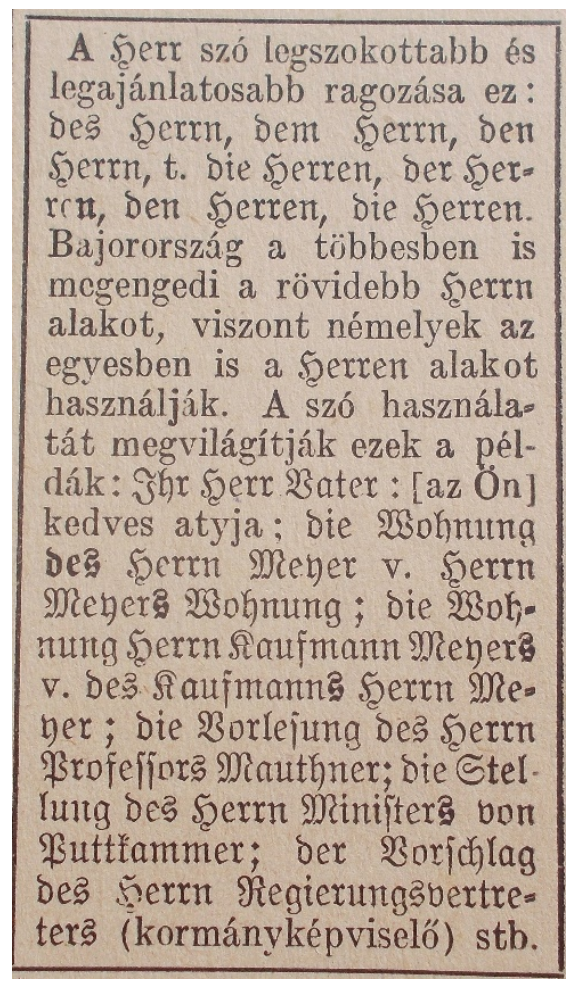

Abbildung 5: Herr (Anwendungsbereich) im KGHDU

Die Metasprache der sprachlichen Informationen im Infokasten ist immer ungarisch.

\section{Innere Mehrsprachigkeit in der Makrostruktur}

\subsection{Orthographie}

In beiden Wörterbüchern wird in den deutschsprachigen Artikelteilen (Stichwort, Angaben zum Stichwort, lexikografische Beispiele) Frakturschrift verwendet. SBDU benutzt deklariert die österreichische Rechtschreibung (SBDU 1899: VII), KGHDU die deutsche (Duden)Rechtschreibung, ohne deutsche Entsprechung stehende österreichische Rechtschreibbesonderheiten gibt es in beiden Wörterbüchern eher selten. Im SBDU wird Früh (als Tageszeit) nach österreichischer Schreibweise als großgeschriebenes Substantiv lemmatisiert (vgl. Abb. 6).

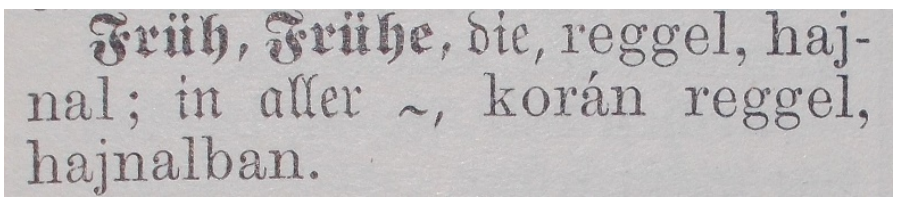

Abbildung 6: Früh als Bezeichnung einer Tageszeit (in Öst.) im SBDU 
Viel häufiger kommt es vor, dass die österreichischen (oder gar bairischen) Entsprechungen im Artikelkopf als Nebenlemmata auf die deutschen Formen (= Hauptlemmata) folgen (vgl. Abb. 7).

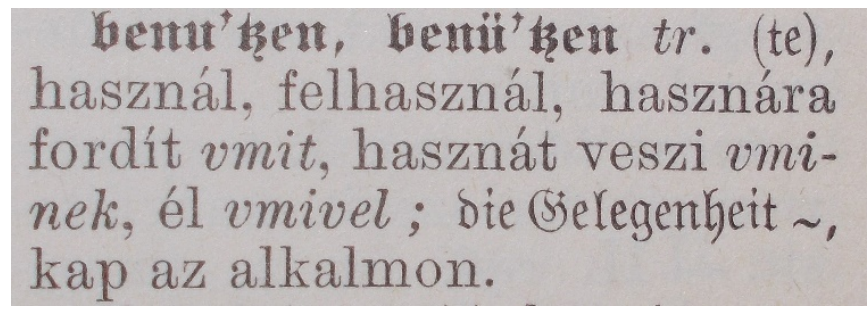

Abbildung 7: benutzen und benützen als Haupt- und Nebenlemma im SBDU

In den Infokästen im KGHDU werden Regeln über und Beispiele für die Vielfalt der regionalen und nationalen Varietäten des Deutschen untergebracht, um den Benutzer gegenüber den unterschiedlichen Sprachnormen zu sensibilisieren. Abb. 8 zeigt einen Infokasten, in dem die Form (und die Schreibweise) jener Pronominaladverbien geklärt wird, die mit hier- beginnen (hierdurch, hierfür, hierher usw.), und es wird darauf verwiesen, dass „die Formen ohne $r$ [...] die Süddeutschen mehr mögen, als die mit einem $r$ [...], wobei in Norddeutschland eher letztere gängig sind“. ${ }^{5}$

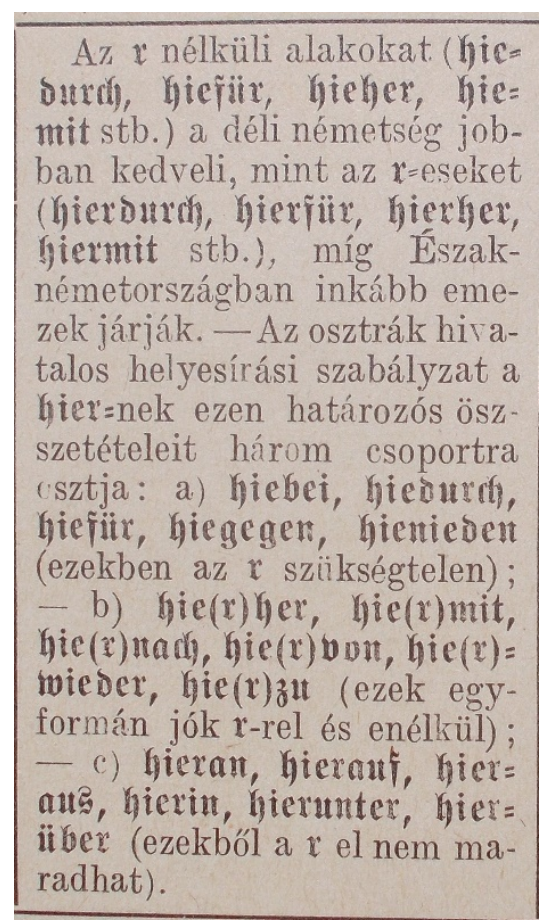

Abbildung 8: Infokasten für die Schreibweise der Pronominaladverbien mit hier- im KGHDU

Ebenda wird die Schreibweise dieser Pronominaladverbien nach dem (damaligen) österreichischen Regelwerk kurz zusammengefasst und mit Beispielen

5 Übersetzung von M. M. 
untermauert, je nachdem, ob diese Pronominalformen im österreichischen Deutsch ein $r$ nicht beinhalten (vgl. Belege in Abb. 8 im Wörterbucheintrag unter Punkt a), ein $r$ beinhalten können (vgl. Belege unter Punkt b, ebd.) sowie für welche das $r$ zwingend notwendig ist (vgl. Belege unter Punkt c, ebd.).

Bei der Gestaltung der orthographischen Informationen stützt sich Kelemen nicht nur auf den - bei der Veröffentlichung des KGHDU bereits auf einem internationalen Konsens ${ }^{6}$ ruhenden - Duden, sondern zieht auch andere Quellen heran, so z.B. das österreichische und das bayerische amtliche Regelwerk (beide aus dem Jahre und Johann Karl Christoph Vogels Schulwörterbuch (1841), wie dies u.a. auch aus den orthographischen Erläuterungen zu dem Stichwort Funke hervorgeht (vgl. Abb. 9):

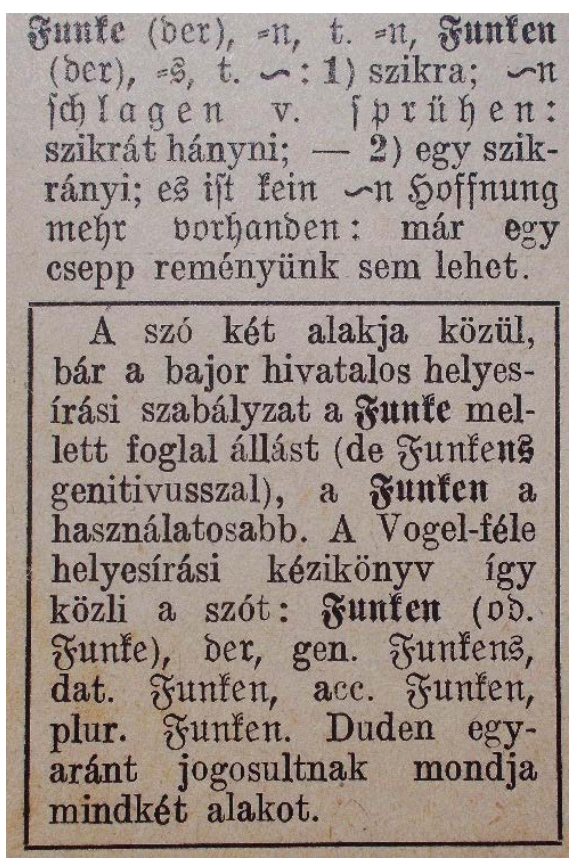

Abbildung 9: Diskussion der Schreibweisen von Funke nach dem bayerischen amtlichen Regelwerk (1879) und Vogel (1841) im KGHD

Da die deutsche Rechtschreibung zum Zeitpunkt der Veröffentlichung des hier untersuchten KGHDU bereits normiert war $^{7}$, ist es nicht verwunderlich, dass orthographische Varianten vor allem im älteren SBDU lemmatisiert wurden: Colonie/Kolonie, Cabinet/Kabinett.

6 Auf der II. Orthographischen Konferenz (1901) wurde die standardisierte deutsche Orthographie von allen deutschsprachigen Ländern angenommen.

7 Die Buchdruckervereine der deutschsprachigen Länder baten Konrad Duden ein Rechtschreibwörterbuch zusammenzustellen, in dem es keine orthographischen Konkurrenzformen gibt. Als Ergebnis ist der Buchdruckerduden (1903) entstanden, der für jedes Wort nur eine Schreibweise zuließ. 


\subsection{Regionalismen}

Der inneren Mehrsprachigkeit wurde in beiden Wörterbüchern auch in der Lemmatisierung von Regionalismen Rechnung getragen: Austriazismen wie Kukuruz, Paradeis, Pomeranze, Topfen kommen in beiden Wörterbüchern als Stichwörter vor. Im KGHDU gab es auch Lemmata, die nur in der österreichischdeutschen Form angegeben sind (Jänner), in der deutschen nicht (*Januar).

Alle zwei Wörterbücher verzeichnen regionale Varianten wie Fleischer / Schlachter / Metzger / Fleischhauer, -hocker, die Angabe der regionalen Gebundenheit des Gebrauchs dieser Wörter fehlt aber im SBDU völlig. Im KGHDU werden vorwiegend die österreichischen und bairischen Formen gekennzeichnet und kommentiert, jedoch auch diese ohne Anspruch auf Vollständigkeit: Kren wird als süddeutsche Form nicht markiert, von seinem Wörterbuchartikel aus wird nicht auf das Lemma Meerrettich (in demselben Wörterbuch) verwiesen. Notabene, zum Eintrag Meerrettich wird ebenfalls nicht hinzugefügt, dass die bairisch-österreichische Entsprechung Kren sei, und ebenso wird auf Kren nicht verwiesen. Dass Kren im süddeutschen Sprachraum benutzt wird $^{8}$ und sein nicht-süddeutsch markiertes Pendant Meerrettich ist, war zu der Zeit der Zusammenstellung der Wörterbuchartikel für die Redakteure offensichtlich nicht relevant, oder in Ermangelung an entsprechenden Referenzwerken (und der dialektologischen Schulung der Verfasser) wurde darauf bewusst verzichtet.

Manche regionalen Varianten erscheinen im Artikelkopf als Nebenlemma, hinter dem Hauptlemma. Letzteres wird der deutschen (Duden)Rechtschreibung entsprechend angesetzt, wie z.B. resch im Artikel resch, rösch im KGHDU (vgl. Abb. 12). Sowohl im SBDU als auch im KGHDU wird von resch auf rösch und umgekehrt verwiesen, die Regionalität der Form rösch durch seine Position als Nebenlemma wird aber nur im KGHDU angedeutet (vgl. Abb. 10, 11, 12 und 13).

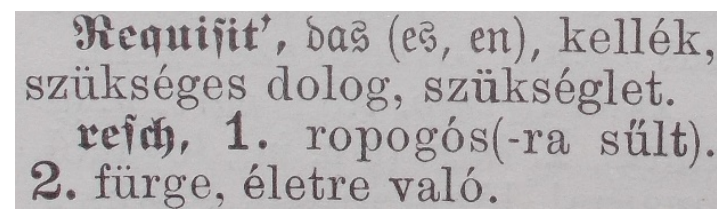

Abbildung 10: resch im SBDU

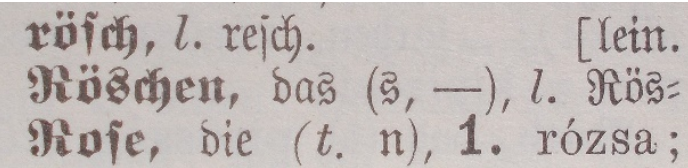

Abbildung 11: rösch im SBDU

8 Die Bezeichnung Kren gelang übrigens durch das Tschechische/Slowakische in die deutsche Sprache in Süddeutschland bzw. Österreich (vgl. Ebner 2008: 16). 


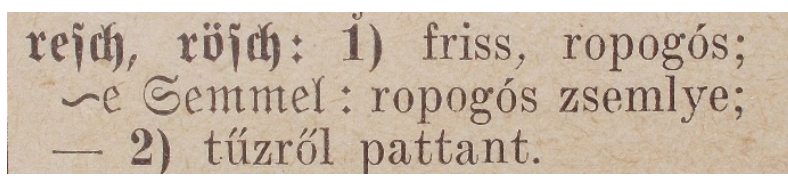

Abbildung 12: resch, rösch im KGHDU

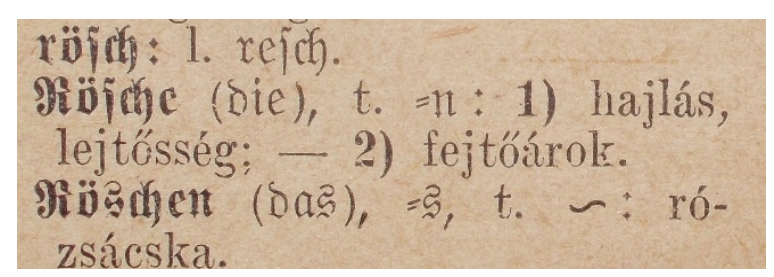

Abbildung 13: rösch im KGHDU

Wissenswertes über die Gebrauchsweisen bestimmter Regionalismen erfährt der Benutzer in dem KGHDU durch die Infokästen. In Bezug auf Lekwar erfährt man durch den angeschlossenen Infokasten, aus welcher Sprache dieses Wort übernommen wurde, dass es nur einem Teil der Österreicher bekannt ist, und dass anstelle von Lekwar in Deutschland Mus verwendet wird (vgl. Abb. 14).

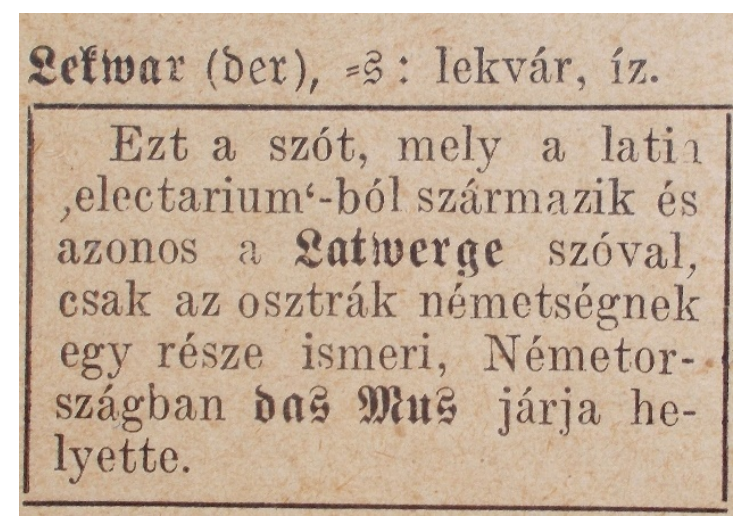

Abbildung 14: Infokasten zum Eintrag Lekwar im KGHDU

Ein Verweis auf das Wort Marmelade, das in demselben Wörterbuch ebenfalls zu finden ist (vgl. Abb. 15), erfolgt nicht, nicht einmal gebracht wird ferner das Synonym Konfitüre. ${ }^{9}$

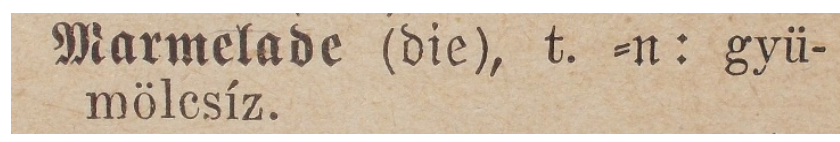

Abbildung 15. Marmelade im KGHDU

9 Die weiteren regional-dialektalen Varianten wie Gsälz, Guts, Schlecksl, Schmärsel, Sießschmeer, Strichi usw. werden in dem KGHDU nicht angegeben, wohl auch aus dem Grund, da einerseits ihre Verbreitung kleinräumiger ist als die von Lekwar, andererseits war Lekwar als Kulturwort auf dem Gebiet der Österreich-Ungarischer Monarchie weit bekannt. 
Das KGHDU achtet auch hinsichtlich der Verbalmorphologie auf oberdeutsche Formen, dem ist es zu verdanken, dass die österreichische Variante röntgenisieren anstelle vom deutschen Pendant röntgen, lemmatisiert ist. ${ }^{10}$ Dasselbe gilt für die Lemmatisierung von Adverbialbildungen: im SBDU finden sich keine Informationen über die österreichischen Formen, wohingegen das KGHDU sie als Stichwort anführt, z.B. weiters.

Der Kommentar der Lemmata in Bezug auf ihre regionale Verbreitung konzentriert sich in erster Linie auf die Erläuterung der oberdeutschen (vornehmlich bairischen und österreichisch-deutschen) Regionalismen. Die Wahl jener süddeutschen Regionalismen, deren Verwendungsgewohnheiten durch einen Infokasten erklärt werden, wurde jedoch willkürlich getroffen.

\section{6 Äußere Mehrsprachigkeit in der Makrostruktur: Internationalismen}

Beide Wörterbücher beinhalten eine Vielzahl an Internationalismen wie z.B. folgende, in beiden Wörterbüchern verzeichnete Entlehnungen: Kassa (it.), Gobelin (frz.), evident (lat.), Partner (eng.), Jause (slowen.), Kren (tschech./slowak.), Magyar (ung.). Die Gebersprachen der Internationalismen sind meistens Französisch, Italienisch, Lateinisch, Englisch, seltener die Sprachen der Österreichisch-Ungarischen Monarchie (Slowenisch, Tschechisch, Slowakisch, Ungarisch). Der Kulturwort-Status dieser Wörter wird grundsätzlich nicht markiert, im SBDU wird bei den Lemmata, bei denen aufgrund der Buchstabenkette eine ungewöhnliche (nicht deutsche) Aussprache zu erwarten ist, die Gebersprache zwar kenntlich gemacht, die ungewöhnliche Artikulation wird aber nicht dargestellt (vgl. Abb. 16).

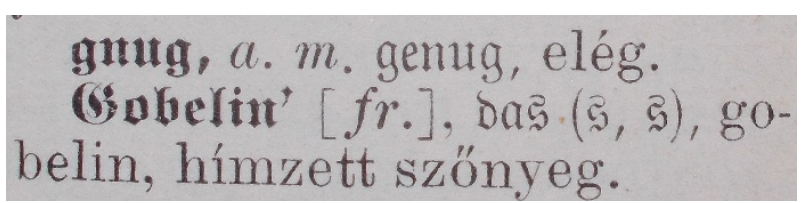

Abbildung 16: Angabe der Wortherkunft von Gobelin im SBDU

Das KGHDU ist hinsichtlich der Herkunft der Internationalismen noch pragmatischer als das SBDU: Grundsätzlich wird sie nicht markiert, dafür folgt bei Stichwörtern mit fremdsprachlicher Artikulation die Angabe der Aussprache mithilfe der Buchstaben des ungarischen Alphabets, ergänzt um ein paar diakritische Zeichen (vgl. Abb. 17).

10 Das Verb röntgen wird von Simonyi / Balassa nicht gebracht, es ist anzunehmen, dass das Tätigkeitswort zu der Zeit der Zusammenstellung der Buchstabenstrecke $R$ weder Simonyi noch Balassa bekannt war, und da die Röntgenstrahlung erst 1895 entdeckt wurde, das Verb auch in den von Simonyi / Balassa als Referenzwerke benutzten Wörterbüchern nicht vorkam. 


\section{Gơbelit [gobleñ]: képszőnyeg. \\ Gispetrie, gen. Sispethes, dat. és}

Abbildung 17. Angabe der Aussprache von Gobelin im KGHDU

\section{Innere Mehrsprachigkeit in der Mikrostruktur}

Wie auf der Makroebene (auf der Ebene der Stichwörter im Wörterverzeichnis), ist die Mehrsprachigkeit auch innerhalb der Mikrostruktur, d.h. innerhalb der Wörterbuchartikel in beiden untersuchten Wörterbüchern präsent, freilich nicht in demselben Maße. Phänomenen der inneren Mehrsprachigkeit (z.B. Hinweise auf österreichische vs. deutsche lexiko-grammatische Merkmale) wird angesichts des primären Charakters eines deutsch-ungarischen Wörterbuchs in den Wörterbucheinträgen mehr Raum geboten als Informationen, die sich auf fremdsprachliche lexiko-grammatische Angaben beziehen.

\subsection{Phonetische Informationen}

Da die Schreibweise der deutschen Wörter (meistens) auf dem Lautprinzip beruht, und in Kenntnis der richtigen Laut-Buchstaben-Zuordnungen die Aussprache des auf Deutsch Geschriebenen für ungarische Benutzer selten eine Fehlerquelle darstellt, beinhaltet weder das SBDU noch das KGHDU eine obligatorische Position für Ausspracheangaben im Artikelkopf. Im KGHDU wird gelegentlich die österreichspezifische Aussprache (hier: Wortakzent) angegeben: Mathematik [...mátik].

\subsection{Morphologische Informationen}

In beiden Wörterbüchern wird auf - in der inneren Mehrsprachigkeit wurzelnde - Genusabweichungen hingewiesen, wenn das Stichwort ein Nomen mit schwankendem Genus ist. In der Auflistung der möglichen Artikelwörter steht im SBDU das deutsche Artikelwort an der ersten Stelle, und das österreichischdeutsche an der zweiten: das, der Polster. Im KGHDU wird die Vorkommenshäufigkeit durch die Abkürzung v. ritkb. ('oder seltener') markiert: das, v. ritkb. der Polster. Kelemen kommentiert die österreichspezifische Genusabweichung auch in Infokästen (vgl. Abb. 18). 


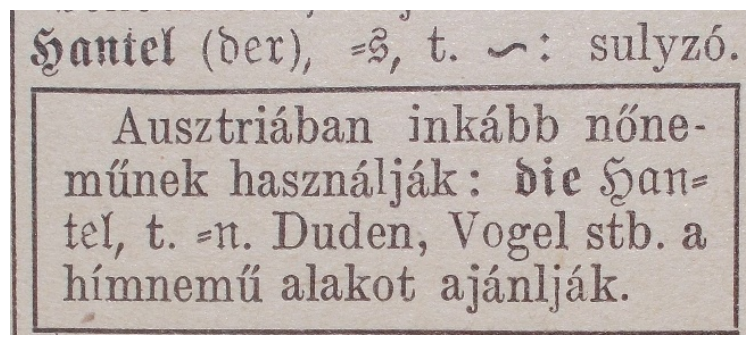

Abbildung 18: Kommentierte Genusabweichung von Hantel im KGHDU

Das SBDU gibt keine Auskunft darüber, dass bestimmte Substantive ihre Mehrzahl mit oder ohne Umlaut bilden können. Das KGHDU merkt dagegen wieder in Infokästen - an, in welchen Regionen mit welcher Bedeutungsmodifikation die Pluralformen mit oder ohne Umlaut gebraucht werden (vgl. Abb. 19).

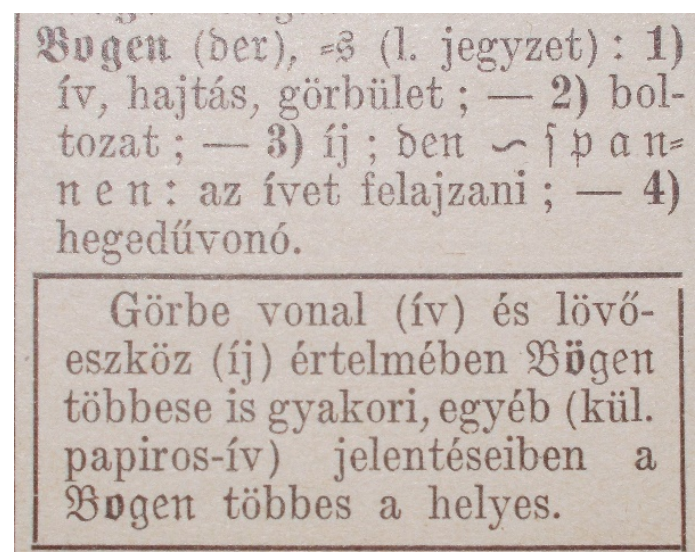

Abbildung 19: Morphosemantischer Kommentar zu Bogen/Bögen im KGHDU

\section{8 Äußere Mehrsprachigkeit in der Mikrostruktur: Phonetische Informationen}

Angaben über die Aussprache von Fremdwörtern, die von der deutschen Hochlautung (im Sinne von Siebs 1898) abweicht, werden in SBDU grundsätzlich mithilfe der Buchstaben des ungarischen Alphabets verschriftet, z.B. Agio [ázsio]. Wenn davon auszugehen ist, dass der Wörterbuchbenutzer imstande ist, den fremdsprachlichen Wortkörper entsprechend den orthographisch-orthoepischen Regeln des Deutschen zu artikulieren, wurde in der Position der Ausspracheangabe nur jener Teil des fremdsprachlichen Wortkörpers mit ungarischen Buchstaben verschriftet, deren Artikulation von der deutschen Hochlautung differiert, z.B. genieren [g=zs]. Im Gegensatz zu Simonyi / Balassi benutzt das KGHDU bei der phonetischen Transkription nichtdeutscher Wortkörper über die Buchstaben des ungarischen Alphabets hinaus auch noch ein paar Sonderzeichen wie z.B. Chaise [sēz], Chansonnette [sanszonëtt] 


\section{Zusammenfassung}

Das Ziel unseres Beitrags war zu zeigen, in welchem Maße zwei deutschungarische Wörterbücher, zwischen deren Erscheinen nur 15 Jahre liegen - das SBDU (1899) und das KGHDU (1914) -, die äußere und innere Mehrsprachigkeit der (gesamt)deutschen Lexik in ihren Makro- und Mikrostrukturen berücksichtigen.

Beide Wörterbücher waren zu ihrer Zeit innovativ, sie haben sich an der ungarischen und deutschen zweisprachigen Lexikografie orientiert, beide wurden von dem Publikum und von Kollegen positiv aufgenommen. Simonyi und Balassa nahmen in ihrer Stichwortliste und in den Wörterbucheinträgen viele Neologismen, Sachmodernismen und Phraseologismen auf. Dass Balassa sein lexikografisches Handwerk gut verstand, davon zeugt auch der Umstand, dass er von Toussaint-Langenscheidt unter Vertrag genommen wurde (vgl. MZsL 1929: 78 sub voce Balassa, 4). Kelemen war gegenüber Neologismen skeptischer als Simonyi / Balassa: Seine Stichwortliste beinhaltet weniger Modernismen, dafür legte er großen Wert auf die Kommentierung der lexikalischen Variation des Deutschen - aus ungarischer Sicht sehr pragmatisch auf die Kommentierung von österreichischen und süddeutschen (bairischen) Bedeutungen und Wortformen. Durch unsere vergleichende Untersuchung konnte festgestellt werden, dass man zur inneren Mehrsprachigkeit bei Kelemen viel mehr Informationen findet als bei Simonyi / Balassa. Diesem benutzerfreundlichen Pragmatismus ist es zu verdanken, dass Kelemen mit seinen zweisprachigen Wörterbüchern unterschiedlichen Formats den ungarischen Markt bis zum Zweiten Weltkrieg dominieren konnte. In der ungarischen Lexikografie wird Kelemen und sein GHWB als Vorläufer der deutsch-ungarischen / ungarisch-deutschen Lexikografie nach 1945 betrachtet.

Die Qualität und die Differenziertheit des KGHWB hängen nicht nur mit der sprachlichen und lexikografischen Kompetenz des Verfassers zusammen: Kelemen war jahrelang auch als Lehrer tätig, und in dieser seiner Qualität konnte er nicht nur die einschlägigen Wörterbücher im Auge behalten, sondern auch die andere Seite der Medaille: auch ihre Benutzer. Sein GHWB genoss wegen seiner Größe und der guten Struktur lange eine Monopolstellung auf dem ungarischen Wörterbuchmarkt.

\section{Literatur}

Bayerisches orthographisches Wörterverzeichnis (1879): München: Verlag von R. Oldenbourg, Abteilung für Schulbücher.

Duden, Konrad (1903): Rechtschreibung der Buchdruckereien deutscher Sprache. Auf Anregung und unter Mitwirkung des Deutschen Buchdruckervereins, des Reichsverbandes Österreichischer Buchdruckereibesitzer und des Vereins Schweizerischer Buchdruckereibesitzer. Leipzig / Wien: Bibliographisches Institut.

Ebner, Jakob (2008): Österreichisches Deutsch. Mannheim / Zürich: Dudenverlag. 
Fejér, Rita (1995): Zur Geschichte der deutsch-ungarischen und ungarisch-deutschen Lexikographie. Von der Jahrhundertwende bis zum Ende des Zweiten Weltkrieges. Tübingen: Max Niemeyer Verlag.

Haarmann, Daniela (2019): Die Ungarische Spracherneuerung. Einleitung in ein Forschungsprojekt. In: Heppner, Harald / Jesner, Sabine (eds.): The 18th Century as Period of Innovation, Yearbook of the Society for 18th Century Studies on South Eastern, 35-49.

Haslinger, Peter (2008): Sprachenpolitik, Sprachendynamik und imperiale Herrschaft in der Habsburgermonarchie 1740-1914. In: Zeitschrift für Osteuropa Forschung (ZfO) 57/1, 81 111.

Kárpáti, Tünde (2003): Vörösmarty német anyanyelvüeknek írt nyelvtana (Kurzgefasste ungarische Sprachlehre für Deutsche) [Die für deutsche Muttersprachler geschriebene Grammatik von Vörösmarty (Kurzgefasste ungarische Sprachlehre für Deutsche)]. In: Hungarológiai évkönyv 4(1). 129-134.

KGHWB = Kelemen, Béla (Hgg.) (1914): Großes Handwörterbuch zur deutschen und ungarischen Sprache mit besonderer Rücksicht auf die Phraseologie. 2. stark vermehrte Auflage. Bd. II, deutsch-ungarischer Teil (nach der neuen Rechtschreibung). Budapest: Verlag der Aktien-Gesellschaft Athenaeum.

MZsL = Újvári, Péter (Hg.) (1929): Magyar Zsidó Lexikon [Ungarisches Jüdisches Lexikon]. Online unter: http://mek-oszk.uz.ua/04000/04093/html/ (05.01.2022)

Österreichisches Wörterbuch (2012). Herausgegeben im Auftrag des Bundesministeriums für Unterricht, Kunst und Kultur; Redaktion: Herbert Fussy, Ulrike Steiner. 42. Auflage. Wien: öbv.

Riehl, Claudia Maria (2014): Mehrsprachigkeit. Darmstadt: Wissenschaftliche Buchgesellschaft.

SBDU = Simonyi, Zsigmond / Balassa, József (Hgg.) (1899): Deutsches und ungarisches Wörterbuch. Erster, deutsch-ungarischer Theil. Budapest: Franklin-Verein.

Siebs, Theodor (1898) (Hg.): Deutsche Bühnenaussprache. Berlin / Köln / Leipzig.

Staatsgrundgesetz vom 21. December [sic] 1867, über die allgemeinen Rechte der Staatsbürger für die im Reichsrathe [sic] vertretenen Königreiche und Länder. Online unter: https://www.ris.bka.gv.at/GeltendeFassung.wxe?Abfrage=Bundesnormen\&Gesetzesnumm er $=10000006(05.01 .2021)$

Vogel, Johann Carl Christoph (1841): Schulwörterbuch der deutschen Sprache. Mit besonderer Rücksicht auf Erleichterung und Förderung der Orthographie. Leipzig: Verlag von Bernhard Tauchnitz jun.

Magyar helyesírás és szóragasztás főbb szabályai [Erste Ungarische Rechtschreibung] (1832). Pest: Magyar Tudós Társaság. 